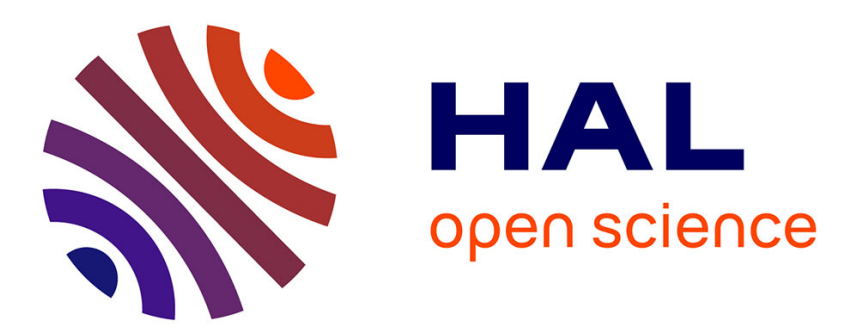

\title{
Unusual internal rotation coupling in the microwave spectrum of pinacolone
}

\author{
Yueyue Zhao, Ha Vinh Lam Nguyen, Wolfgang Stahl, Jon T Hougen
}

\section{To cite this version:}

Yueyue Zhao, Ha Vinh Lam Nguyen, Wolfgang Stahl, Jon T Hougen. Unusual internal rotation coupling in the microwave spectrum of pinacolone. Journal of Molecular Spectroscopy, 2015, 318, pp.91-100. 10.1016/j.jms.2015.10.005 . hal-03183156

\section{HAL Id: hal-03183156 https://hal.science/hal-03183156}

Submitted on 26 Mar 2021

HAL is a multi-disciplinary open access archive for the deposit and dissemination of scientific research documents, whether they are published or not. The documents may come from teaching and research institutions in France or abroad, or from public or private research centers.
L'archive ouverte pluridisciplinaire HAL, est destinée au dépôt et à la diffusion de documents scientifiques de niveau recherche, publiés ou non, émanant des établissements d'enseignement et de recherche français ou étrangers, des laboratoires publics ou privés. 


\title{
Unusual internal rotation coupling in the microwave spectrum of pinacolone
}

\author{
Yueyue Zhao ${ }^{a}$, Ha Vinh Lam Nguyen ${ }^{\star b}$, Wolfgang Stahla, and Jon T. Hougen ${ }^{c}$ \\ ${ }^{a}$ Institute of Physical Chemistry, RWTH Aachen University, Landoltweg 2, D-52074 Aachen, Germany \\ ${ }^{b}$ Laboratoire Interuniversitaire des Systèmes Atmosphériques (LISA), UMR 7583 (CNRS/Univ. Paris Est \& Paris \\ Diderot), Université Paris Est, 61 avenue du Général de Gaulle, F-94010 Créteil cedex, France \\ ${ }^{c}$ Sensor Science Division, National Institute of Standards and Technology, Gaithersburg, MD, 20899-8441, USA
}

* Corresponding Author: Dr. Ha Vinh Lam Nguyen, Email: lam.nguyen@lisa.u-pec.fr, Tel.: 0033145176553.

The molecular beam Fourier-transform microwave spectrum of pinacolone (methyl tert-butyl ketone) has been measured in several regions between 2 and $40 \mathrm{GHz}$. Fits of the assigned spectrum using several computer programs based on different models for treating torsion-rotation interaction lead to the conclusion that no existing program correctly captures the internal dynamics of this molecule. Quantum chemical calculations at the MP2/6-311++G(d,p) level of theory indicate that this molecule does not have a plane of symmetry at equilibrium, and that internal rotation of the light methyl group induces a large oscillatory motion of the heavy tert-butyl group from one side of the $\mathrm{C}_{\mathrm{s}}$ configuration to the other. This effect has been modeled for $J=0$ levels by a relatively simple two-top torsional Hamiltonian, where the magnitudes of the strong coupling terms between the tops are determined directly from the ab initio two-dimensional potential surface. A plot of the resultant $0 \mathrm{~A}, 0 \mathrm{E}, 1 \mathrm{E}, 1 \mathrm{~A}$ torsional levels on the same scale as a one-dimensional potential curve along the zig-zag path connecting the six (unequally spaced) minima bears a striking resemblance to the 1:2:1 splitting pattern of the A, E, E, B levels of an internal rotation problem with a six-fold barrier. It seems likely that rotational transitions within the $1 \mathrm{E}$ and $1 \mathrm{~A}$ torsional levels are the cause of the roughly $50 \%$ of the spectrum that remains unassigned after all predicted transitions within the $0 \mathrm{~A}$ and $0 \mathrm{E}$ torsional levels are removed. However, a much more complete measurement campaign and some new torsion-rotation theory will be needed to verify this hypothesis.

\section{Introduction}

Pinacolone (methyl tert-butyl ketone or 3,3-dimethyl-2-butanone), with the structure shown in Fig. 1, is a molecule of chemical, spectroscopic, and quantum chemical interest.

Chemically, pinacolone belongs to the class of small ketones, which are widely used industrially as solvents and as reactants in syntheses. In addition, small ketones and small esters taken together contain a number of common odorant molecules and some pheromones. Many of these have been the object of microwave studies in the Aachen group, for example 
in Refs. [1-4] for esters and in Refs. [5-7] for ketones, with a view toward determining conformational and dynamical properties that might eventually be correlated with processes involved in the sense of smell. Rotational spectra of similar esters and ketones have also been investigated in other groups [8,9]. Pinacolone itself has a camphor or peppermint-like smell. Its vapor pressure of about $4 \mathrm{kPa}$ at $25^{\circ} \mathrm{C}$ allows easy microwave observations at room temperature or with molecular beam techniques.

\section{Figure 1:}

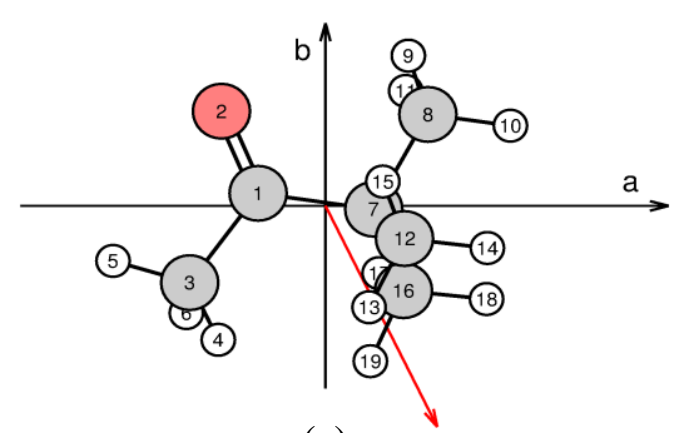

(a)

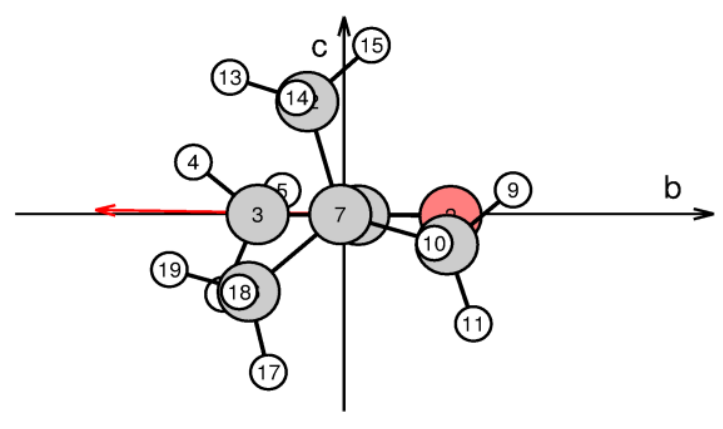

(b) The atom numbering and structure

of pinacolone and its dipole moment (in red), as viewed along two of its principal axes. (a) View from the positive $c$ axis, illustrating: (i) a classic ketone structure, (ii) a methyl-top axis nearly bisecting the $a$ and $b$ directions, and (iii) a dipole moment almost exactly parallel to the $\mathrm{C}=\mathrm{O}$ bond. (b) View from the positive $a$ axis, showing that: (i) the $a$ axis is nearly coincident with the $\mathrm{C}_{7}-\mathrm{C}_{1}$ bond, (ii) the four atoms $\mathrm{C}_{3}$, $\mathrm{C}_{7}, \mathrm{C}_{1}, \mathrm{O}_{2}$ lie almost exactly in the $a b$ plane, and (iii) the plane of symmetry of the tert-butyl group is rotated about $16^{\circ}$ out of the $\mathrm{C}_{3}, \mathrm{C}_{7}, \mathrm{C}_{1}$, $\mathrm{O}_{2}$ plane. This structure can potentially exhibit distortions and/or large-amplitude motions associated with internal rotation of the acetyl methyl group, internal rotation of the tert-butyl group, or out-of-plane motions of the $\mathrm{C}_{3}, \mathrm{C}_{7}, \mathrm{C}_{1}, \mathrm{O}_{2}$ atoms.

Spectroscopically, pinacolone is a close relative of acetone, where one of the two equivalent $C_{3 v}$ methyl groups has been replaced by the much heavier and much bulkier tert-butyl group. From the point of view of its large amplitude motions, pinacolone has a simple $\mathrm{C}_{3 \mathrm{v}}$ methyl rotor on one side of the carbonyl group (called the acetyl methyl group from now on) and ${ }_{a} \mathrm{C}_{3 v}$ tert-butyl rotor on the other side, which itself contains three $\mathrm{C}_{3 \mathrm{v}}$ methyl rotors. When starting this work, we expected the spectrum of pinacolone to be essentially that of a one-methyl-top molecule for the following reasons: (i) splittings arising from internal rotation of the whole tert-butyl group should be quite small, since its moment of inertia is about 20 times larger than that of a methyl group, and (ii) splittings arising from internal rotation of the three individual methyl groups within the tertbutyl moiety should also be quite small, since the barriers to such motions are expected to be approximately $1000 \mathrm{~cm}^{-1}$, similar to the barrier of the methyl group in ethyl chloride [10]. However, as we shall see below, this simple one-top expectation turned out to be completely incorrect. 
Quantum chemically, the relatively small empirical formula of $\mathrm{C}_{6} \mathrm{H}_{12} \mathrm{O}$ suggests that the size of the molecule is well within modern computational capabilities. However, in some ketones we studied before, like methyl isobutyl ketone [6] or methyl neopentyl ketone [7], small deviations from high-symmetry assumptions were found in the calculated structures. Small calculated deviations are not always easy to believe when they are based on the rather modest quantum chemistry calculations carried out in experimental spectroscopic laboratories and when they conflict with chemical intuition. Furthermore, the presence of small deviations from high-symmetry equilibrium geometries is frequently difficult to demonstrate experimentally, especially when zero-point motions are large enough to mask the spectroscopic effects of these small deviations. As the present study unfolded, however, we encountered a number of spectroscopic difficulties, leading us to suspect that such deviations are, in fact, present in pinacolone. For example we now believe that: (i) pinacolone may not have a plane of symmetry in its equilibrium configuration, and (ii) the barrier to internal rotation of the acetyl methyl group $\mathbf{C H}_{3}-(\mathrm{C}=\mathrm{O})-\mathrm{C}(\mathrm{CH})_{3}$ may not be well approximated by a single $\cos 3 \alpha$ term.

\section{Quantum chemical calculations}

\subsection{Geometry optimization}

In pinacolone, rotation around the $\mathrm{C}_{1}-\mathrm{C}_{3}$ bond (for atom numbering see Fig. 1) by varying the dihedral angle $\alpha_{1}=\angle\left(\mathrm{O}_{2}\right.$, $\mathrm{C}_{1}, \mathrm{C}_{3}, \mathrm{H}_{5}$ ) corresponds to internal rotation of the acetyl methyl group and the rotations around the $\mathrm{C}_{7}-\mathrm{C}_{8}, \mathrm{C}_{7}-\mathrm{C}_{12}$, and $\mathrm{C}_{7}-\mathrm{C}_{16}$ bonds correspond to internal rotations of the three tert-butyl methyl groups. We thus created four starting geometries by varying only the dihedral angle $\alpha_{2}=\angle\left(\mathrm{O}_{2}, \mathrm{C}_{1}, \mathrm{C}_{7}, \mathrm{C}_{8}\right)$ in a grid of $30^{\circ}$, corresponding to a rotation around the $\mathrm{C}_{1}-\mathrm{C}_{7}$ bond. Quantum chemical optimizations at the MP2/6-311++G(d,p) level of theory using the program GAUSSIAN 09 [11,12] yielded only one stable conformer (shown in Fig. 1), with rotational constants $A=3.1881 \mathrm{GHz}, B=2.3149 \mathrm{GHz}$, and $C=1.9223 \mathrm{GHz}$, and dipole moment components $\mu_{\mathrm{a}}=1.47 \mathrm{D}, \mu_{\mathrm{b}}=-3.03 \mathrm{D}$, and $\mu_{\mathrm{c}}=0.05 \mathrm{D}$. The Cartesian coordinates are given in Table S-1 in the supplementary material.

The dihedral angle $\alpha_{2}$ of the optimized geometry in Fig. 1 is surprisingly not $0^{\circ}$ as expected for $\mathrm{C}_{\mathrm{s}}$ symmetry, i.e. the tertbutyl group does not share a plane of symmetry with the $\mathrm{CH}_{3}-\mathrm{C}=\mathrm{O}$ frame. This type of symmetry breaking has frequently occured in quantum chemical calculations on other ketones studied in Aachen. For example, a tilt angle out of the $\mathrm{C}-(\mathrm{C}=\mathrm{O})-\mathrm{C}$ plane of about $10^{\circ}$ was calculated for each ethyl group in diethyl ketone [5]. A similar tilt angle was found for the tert-butyl group in methyl neopentyl ketone [7], a close relative of pinacolone where the tert-butyl group is one carbon atom further from 
the carbonyl group. On the other hand, for the isopropyl group in methyl isobutyl ketone [6], a tilt angle of approximately 20 ${ }^{\circ}$ was calculated.

\subsection{Methyl Internal Rotation}

For quantum chemical study of internal rotation, the acetyl methyl group was rotated by varying the dihedral angle $\alpha_{1}$ in $10^{\circ}$ steps from $-60^{\circ}$ to $60^{\circ}$. Due to the $C_{3 v}$ symmetry of the methyl group, only a $120^{\circ}$ rotation of $\alpha_{1}$ was sufficient. All other structural parameters were optimized. By checking the optimized $\alpha_{2}$ dihedral angle for various fixed $\alpha_{1}$ values (and as might be expected from the presence of the $10^{\circ}$ tilt angle in the equilibrium structure), it became clear that quantum chemical results at our level of calculation indicated that these two internal rotations are strongly correlated, and that rotation of the methyl group forces an oscillation of the tert-butyl group. As a consequence, the potential energy function for the acetyl methyl group internal rotation cannot be well represented by a dominant $V_{3}$ term.

In spite of these quantum chemistry indications of potential trouble, we began our spectroscopic analysis, as described in section 3, under the assumption that effects from a slightly distorted, low-symmetry equilibrium configuration could be essentially ignored, either because they were intrinsically small, or because zero-point vibrations made them small. When all attempts at obtaining a good fit to the spectral measurements failed, we looked again at our quantum chemistry calculations. The two-dimensional potential surface describing this coupled motion and its effects on the torsion-rotation energy levels and wave functions will be discussed in detail in Section 4. For comparison with the traditional fits of the microwave spectrum described in Section 3, we note that the acetyl methyl internal-rotation barrier height predicted from our quantum chemistry calculations is $156 \mathrm{~cm}^{-1}$.

\section{Microwave spectroscopy}

\subsection{Measurements}

Pinacolone was purchased from Merck Schuchardt, Hohenbrunn, Germany [12], and used without further purification. Two molecular beam Fourier transform microwave spectrometers, in the frequency ranges from 2 to $26.5 \mathrm{GHz}$ [13] as well as 26.5 to $40 \mathrm{GHz}$ [14], were used. A mixture of $1 \%$ by volume of pinacolone in helium at a total pressure of 1 bar was expanded through a pulsed nozzle into the vacuum chamber, with a pressure of $10^{-6}$ mbar. The rotational temperature was approximately $2 \mathrm{~K}$. The measurements were carried out first in a rapid-scan mode, where overlapping spectral segments were recorded with a step size of $0.25 \mathrm{MHz}$. All lines from the scans were remeasured in the high-resolution mode, with a measurement accuracy of $2 \mathrm{kHz}$ (type $\mathrm{B}, k=1[15])$. 
The measurements for this molecule are significantly more extensive than usual, because questions associated with the adequacy of the effective Hamiltonian used to fit the observed frequencies made it important to establish the correctness of as many assignments as possible by putting transitions into combination difference loops (also called Ritz cycles), as discussed in more detail below. Figs. 2 and 3, respectively, show schematic plots of the energies of the A and E rotational levels of pinacolone (open circles) organized into columns of increasing $J$ and given $K_{a}$, for $0 \leq K_{a} \leq 6$ and $K_{a} \leq J \leq 10$. Lines connecting pairs of circles indicate transitions measured in the present work. It can be seen that the vast majority of assignments are supported by Ritz cycles, whose frequency sums (not shown) equal zero to within 4 or $5 \mathrm{kHz} \approx \sqrt{4} \times$ (measurement error).

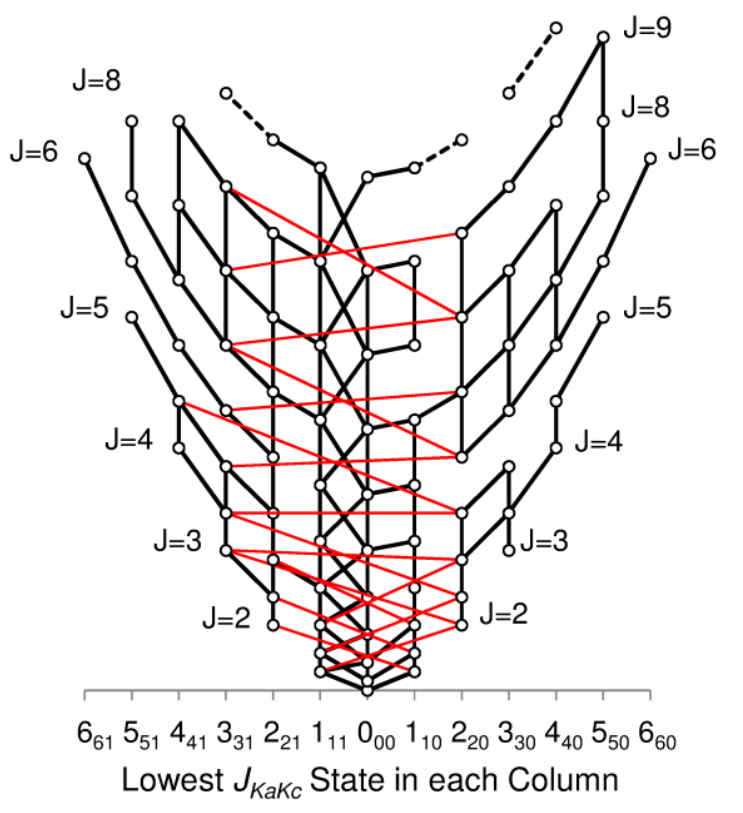

Figure 2: Schematic illustration of almost all the A species rotational energy levels of pinacolone observed in this work (open circles). Solid lines connecting the circles indicate transitions that were included in the RAM36 fit given in the supplementary file. Dashed lines indicate a few transitions that were assigned but not included in that fit. Energy levels are plotted in columns labeled by a given $K_{a}$, for the range $0 \leq$ $K_{a} \leq 6$. The levels in each column increase in $J$ from $J=K_{a}$ to $J=11$. For pairs of columns with the same $K_{a}$ but different $K_{c}$, the column with $K_{a}+K_{c}=J+1$ is plotted to the left of the central $K_{a}=0$ position, while the column with $K_{a}+K_{c}=J$ is plotted to the right. The vertical black lines are $\Delta K_{a}=0$ transitions; the black lines falling on "parabolas" are $\Delta J=0$ transitions. The thin red lines are transitions that connect levels on the left of this diagram with levels on the right. It can be seen that assignments for a large number of lower- $J$ transitions can be checked by combination difference loops, which sum to values in good agreement with the expected measurement uncertainty of $2 \mathrm{kHz}$. Slightly over 40 assigned and fitted A species transitions are not shown in this figure to avoid clutter. Many of them connect states to the left of the $K_{a}=0$ column with states to the right, and make combination difference loops possible for a number of additional transitions at the edges of this figure. 


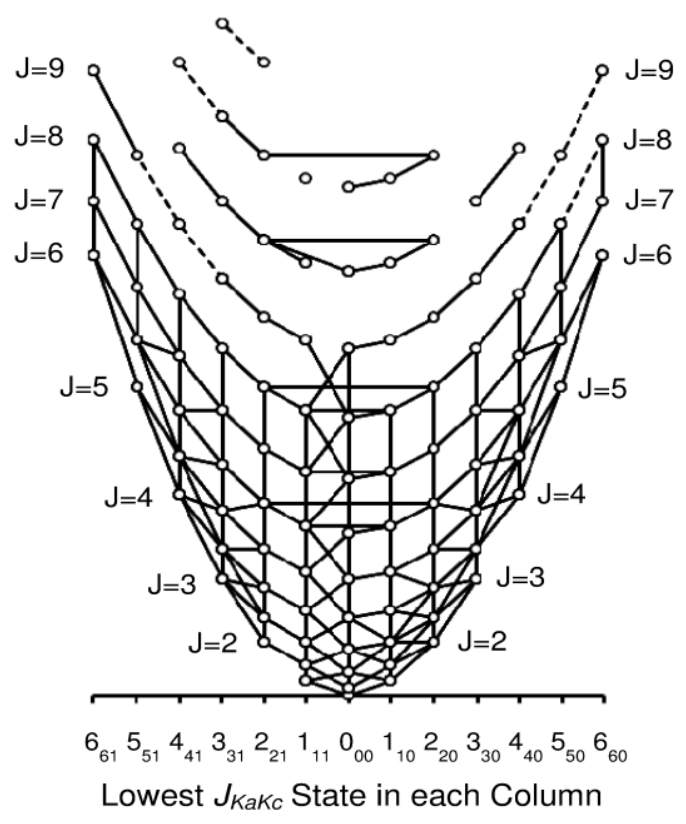

Figure 3: Schematic illustration of the E species rotational energy levels of pinacolone observed in this work, using the same general layout as in Fig. 2. It can be seen that assignments for the vast majority of $J^{\prime}-J^{\prime \prime}$ transitions with $J^{\prime} \leq 8$ can be checked by combination difference loops, which again sum to values in good agreement with the measurement uncertainty. Slightly more than 30 assigned and fitted transitions connecting states on the left and right of the figure (analogous to the red lines in Fig. 2) are not shown. They confirm many existing combination-difference loops, but add no new ones.

\subsection{Assignments}

The initial assignment procedure is described in more detail in Ref. 16. Briefly, as a first step, the theoretical pinacolone spectrum was calculated using rotational constants obtained from quantum chemical calculations at the MP2/6-311++G(d,p) level of theory (see section 2.1). From the calculated dipole moment components $\mu_{\mathrm{a}}=1.47 \mathrm{D}, \mu_{\mathrm{b}}=-3.03 \mathrm{D}$, and $\mu_{\mathrm{c}}=0.05 \mathrm{D}$, only $a$ - and $b$-type lines were predicted and the $b$-type lines are expected to be much stronger. At this stage, 372 transitions were found in the discontinuous scans from 8.0 to $40 \mathrm{GHz}$. As the second step, a number of possible Ritz cycles were identified in the observed frequency list. The deviations of the four-line frequency sums in all cycles agreed perfectly with the presumed experimental measurement uncertainty of $2 \mathrm{kHz}$. By comparing the predicted and experimental spectrum and using information from the Ritz cycles, many lines of A species in the scans could be assigned, yielding rotational and centrifugal distortion constants that allowed reliable predictions. Totally, 160 A species lines could be fitted with the program XIAM [17] to a standard deviation of $15.6 \mathrm{kHz}$, which is much greater than the experimental accuracy of $2 \mathrm{kHz}$ that we often achieve by fitting separately the A species of other molecules with internal rotation. 
As a next step, the barrier to internal rotation was assumed to be that of acetone $\left(265 \mathrm{~cm}^{-1}\right)$. A torsion-rotation spectrum with $\mathrm{A}$ and $\mathrm{E}$ species was predicted using the program XIAM. The angles between the internal rotor axis and the principal axes were taken from the geometry given by the quantum chemical calculations described in section 2.1 . Trial and error E species assignments led to no success, so we decided to vary the barrier. At a barrier of about $122 \mathrm{~cm}^{-1}, 41 \mathrm{E}$ species lines could be fitted. We were quite confident that these E species assignments were correct, since they could all be confirmed by Ritz cycles, and we thus expected to use predictions from this fit to find new lines outside of the already scanned regions by carrying out small scans of about $5 \mathrm{MHz}$. Surprisingly, we were not able to find any lines near the predicted frequencies.

\subsection{Fits}

We now consider a number of fitting models traditionally applied to the microwave spectra of asymmetric top molecules with one methyl rotor. Since none of these models explicitly allow for the coupling between internal rotation of the methyl and tert-butyl tops predicted by our quantum chemical results, we are in some sense testing whether the effects of this coupling prevent a normal torsion-rotation fitting treatment.

\subsubsection{XIAM fits}

The results of a XIAM fit of 160 A species lines using three rotational constants, five quartic, and four of six sextic centrifugal distortion constants, giving a standard deviation of $15.6 \mathrm{kHz}$, are shown in Table 1 . A global fit of 160 A species and $41 \mathrm{E}$ species transitions is also given in Table 1, and yielded a barrier to internal rotation of $121.532(57) \mathrm{cm}^{-1}$. The angle between the internal rotor axis and the $c$ principal axis was fixed to $90^{\circ}$, since an effective $\mathrm{C}_{\mathrm{s}}$ geometry was assumed. The angles to the $a$ and $b$ principal axes, as well as two higher order parameters $\mathrm{D}_{\mathrm{pi} 2 J}$ and $\mathrm{D}_{\mathrm{pi} 2-}$, were fitted. The standard deviation was 1.16 MHz, which is nearly 600 times the experimental accuracy of $2 \mathrm{kHz}$. Many centrifugal distortion constants could not be well determined because of this huge standard deviation, and thus had to be fixed at values taken from the A species fit. All fitted transitions are listed in Table S-2. The barrier of $122 \mathrm{~cm}^{-1}$ determined above is relatively low and from our experience, the program XIAM can often not fit microwave spectra to experimental accuracy for molecules with an internal rotation barrier this low. Since no further predicted lines could be found, we decided to use other programs (which use other formalisms to treat the internal rotation) in order to check the assignments and find new lines.

\subsubsection{ERHAM fits}

The $160 \mathrm{~A}$ and $41 \mathrm{E}$ species lines assigned in the XIAM Fit A-E in Table 1 were put into the program ERHAM [19], leading to a total number of $168 \mathrm{~A}$ and $43 \mathrm{E}$ fitted transitions. All transition frequencies together with their residual deviations are also 
in Table S-2. We found that 8 additional A species lines, but only two more E species lines could be assigned, even though the standard deviation of the ERHAM fit decreases to $10.6 \mathrm{kHz}$, which is still higher than the experimental accuracy, but already a dramatic improvement from the deviation of $1.16 \mathrm{MHz}$ obtained by the program XIAM. However, a number of effective parameters were added in the ERHAM fit, as shown in Table S-3. The comparable parameters in XIAM and ERHAM are given in Table 1.

Even though the standard deviation of the ERHAM fit was only $10.6 \mathrm{kHz}$, no further E species lines could be included in the fit, whereas over 150 strong lines in the scans remained unassigned. By this stage, we suspected that an extension of the internal rotation model was needed to include, for example, additional parameters which could implicitly take into account the coupling between the acetyl methyl and the tert-butyl group internal rotations. Furthermore, the large moment of inertia of the tert-butyl group compared to that of the methyl rotor suggests that small structural changes often occurring during internal rotation motion might have effects on the rotational energy levels that are much larger than normal.

\subsubsection{BELGI-C $C_{s}$ fits}

As indicated above, there is some reason to worry that small structural changes during methyl top rotation could lead to larger than normal torsion-rotation interactions in pinacolone. However, from our chemical intuition, we still assumed an effective $\mathrm{C}_{\mathrm{s}}$ structure and fixed the angle between the internal rotor axis and the $c$ principal axes $\angle(\mathrm{i}, c)$ to $90^{\circ}$ in both the XIAM and

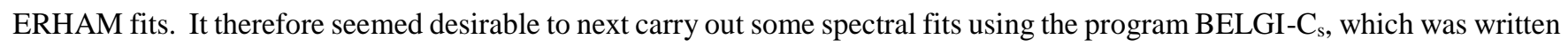
for one-top molecules with a plane of symmetry at equilibrium [20]. Even though this program is written in a coordinate system based on the concept of a rigid top rotating against a rigid frame, it contains a rather large number of symmetry-allowed higherorder terms which take into account in a phenomenological fashion a variety of torsion-rotation interaction effects.

Using the program BELGI-C $\mathrm{s}_{\mathrm{s}}$ available at PROSPE [21], a fit with 31 adjustable parameters was gradually achieved for a data set containing 174 A lines and 156 E lines. Parameters that can be transformed into the PAM system and then compared with the corresponding parameters from XIAM are given in Table 1 . The complete parameter set in the RAM system is available in the supplementary material (Table S-4); all fitted transitions are listed in Table S-5. Unfortunately, the root-mean-square (rms) deviations for the set of A species lines was $28 \mathrm{kHz}$, while that for the E species lines was $64 \mathrm{kHz}$. These rms deviations are approximately 15 and 30 times larger, respectively, than the measurement precision of about $2 \mathrm{kHz}$.

Attempts to reduce these rms deviations were not successful. We thus began to speculate that the cause of our fitting troubles lay in the fact that BELGI-C $\mathrm{S}_{\mathrm{s}}$ contains mainly those high-order terms that are expected to be large when the top axis lies nearly 
along the principal $a$ axis, whereas in pinacolone, the symmetry axis of the methyl top lies approximately halfway between the principal $a$ and $b$ axes, as can be seen from Table 1 . 
Table 1. The molecular parameters of pinacolone from the fits using different programs and from quantum chemical calculations.

\begin{tabular}{|c|c|c|c|c|c|c|c|c|}
\hline Constant $^{\mathrm{a}}$ & Unit & $\begin{array}{c}\text { XIAM } \\
\text { Fit A }\end{array}$ & $\begin{array}{l}\text { XIAM } \\
\text { Fit A-E }\end{array}$ & ERHAM & BELGI-Cs & RAM36 & BELGI-C $_{1}$ & Calc. $^{b}$ \\
\hline$A$ & $\mathrm{GHz}$ & $3.1920821(18)$ & $3.18450(21)$ & $3.1890666(87)$ & $3.490(19)$ & $3.2975(64)$ & $5.266(89)$ & 3.188 \\
\hline$B$ & $\mathrm{GHz}$ & $2.29289262(56)$ & $2.28129(37)$ & $2.2905623(41)$ & $2.271(16)$ & $1.8517(68)$ & $4.522(89)$ & 2.315 \\
\hline$C$ & $\mathrm{GHz}$ & $1.92818626(39)$ & $1.93052(17)$ & $1.9282594(22)$ & $1.9189(20)$ & $1.7368(39)$ & $4.095(89)$ & 1.922 \\
\hline$\Delta_{\mathrm{J}}$ & $\mathrm{kHz}$ & $-1.6188(43)$ & $-1.10(18)$ & $-4.615(69)$ & $-59.2(37)$ & 0. (fixed) & $-677(55)$ & \\
\hline$\Delta_{\mathrm{JK}}$ & $\mathrm{kHz}$ & $92.227(50)$ & $92.32(97)$ & $109.7(10)$ & & & & \\
\hline$\Delta_{\mathrm{K}}$ & $\mathrm{kHz}$ & $-90.16(16)$ & $-87.9(20)$ & $-81.77(98)$ & & & & \\
\hline$\delta \mathrm{J}$ & $\mathrm{kHz}$ & $0.2076(23)$ & $0.2076^{\mathrm{c}}$ & $-0.537(41)$ & & & & \\
\hline$\delta_{\mathrm{K}}$ & $\mathrm{kHz}$ & $-254.617(36)$ & $-254.82(89)$ & $-342.43(73)$ & & & & \\
\hline$\Phi_{\mathrm{JK}}$ & $\mathrm{kHz}$ & $-0.0711(11)$ & $-0.0711^{\mathrm{c}}$ & $-0.299(23)$ & & & & \\
\hline$\Phi_{\mathrm{KJ}}$ & $\mathrm{kHz}$ & $0.1496(33)$ & $0.1496^{\mathrm{c}}$ & & & & & \\
\hline$\Phi_{\mathrm{K}}$ & $\mathrm{kHz}$ & $-0.0780(43)$ & $-0.0780^{c}$ & & & & & \\
\hline$\phi_{\mathrm{K}}$ & $\mathrm{kHz}$ & $-0.4142(42)$ & $-0.4142^{c}$ & & & & & \\
\hline$\phi_{\mathrm{JK}}$ & & & & $-0.131(14)$ & & & & \\
\hline$V_{3}$ & $\mathrm{~cm}^{-1}$ & & $121.532(57)$ & & $90.53(76)$ & $112.07(56)$ & $99.71483(30)$ & 156 \\
\hline$I_{\alpha}$ & $\mathrm{u} \AA^{2}$ & & $3.1986^{\mathrm{d}}$ & $2.635(23)$ & $3.3113(2)^{\mathrm{e}}$ & $3.2993(2)^{\mathrm{e}}$ & $3.3531(2)^{\mathrm{f}}$ & \\
\hline$\angle(\mathrm{i}, a)$ & $\circ$ & & $50.063(51)$ & $34.62(35)$ & $29.05(80)^{\mathrm{e}}$ & $41.23(35)^{\mathrm{e}}$ & $36.5(44)^{\mathrm{f}}$ & 53.7 \\
\hline$\angle(\mathrm{i}, b)$ & $\circ$ & & $39.937(51)$ & $55.38(35)$ & $60.94(80)^{e}$ & $48.77(35)^{\mathrm{e}}$ & $53.5(44)^{f}$ & 36.3 \\
\hline$\angle(\mathrm{i}, c)$ & $\circ$ & & $90.0^{\mathrm{g}}$ & $90.0^{\mathrm{g}}$ & $90.0^{\mathrm{g}}$ & $90.0^{\mathrm{g}}$ & $90.293(97)^{\mathrm{f}}$ & 89.7 \\
\hline $\mathrm{D}_{\mathrm{pi} 2 J}$ & $\mathrm{MHz}$ & & $0.562(37)$ & & & & & \\
\hline $\mathrm{D}_{\mathrm{pi2}-}$ & $\mathrm{MHz}$ & & $0.994(38)$ & & & & & \\
\hline$\sigma_{\mathrm{A}} / \sigma_{\mathrm{E}}{ }^{\mathrm{h}}$ & $\mathrm{kHz}$ & $15.6 /-$ & 1155.8 & 10.6 & $28.2 / 63.5$ & $8.8 / 21.4$ & $85.5 / 129.6$ & \\
\hline $\mathrm{N}_{\mathrm{A}} / \mathrm{N}_{\mathrm{E}}{ }^{\mathrm{i}}$ & & $160 / 0$ & $160 / 41$ & $168 / 43$ & $174 / 156$ & $174 / 228$ & $124 / 174$ & \\
\hline
\end{tabular}

a All constants refer to the principal inertial axis system. For the centrifugal distortion constants shown, Watson's A reduction and Ir representation were used [18]. Centrifugal distortion constan
for the BELGI and RAM36 fits are not shown, because they cannot easily be converted from RAM to PAM values. Parameters are given with one standard uncertainty in parentheses (type A,

$k=1[15])$, unless otherwise indicated.

${ }^{\mathrm{b}}$ Calculation at the MP2/6-311++G(d,p) level of theory.

${ }^{\mathrm{c}}$ Fixed to the values from XIAM Fit A (see text).

${ }^{\mathrm{d}}$ Moment of inertia of the internal rotor, derived from $F_{0}=158 \mathrm{GHz}$ (fixed).

${ }^{\mathrm{e}}$ Derived from the fitted RAM constants A, B, C, DAB and the fixed value $F=5.2 \mathrm{~cm}^{-1}$ (rather than from the fitted constants A, B, C, DAB, and $\rho$ ). Uncertainties in parentheses are type B, $k=1$ [15].

${ }^{\mathrm{f}}$ Derived from the fitted RAM constants A, B, C, DAB, DACI and the fixed value $F=5.2 \mathrm{~cm}^{-1}$. Uncertainties in parentheses are type B, $k=1[15]$.

${ }^{\mathrm{g}}$ Fixed due to the assumed $\mathrm{C}_{\mathrm{s}}$ symmetry.

${ }^{\mathrm{h}}$ Standard deviation of the fit, or root-mean-square deviations for the A and the E species individually when separated by a slash (/).

${ }^{\mathrm{i}}$ Number of the fitted $\mathrm{A}$ and $\mathrm{E}$ species transitions 


\subsubsection{RAM36 fits}

We thus switched at this point to the program RAM36 [22] (also available from PROSPE [21]), which allows the user to choose his own set of symmetry-allowed torsion-rotation interaction terms for use in the fitting Hamiltonian. This feature allowed us to experiment with a number of infrequently used higher-order rotational and torsional centrifugal distortion corrections to various simple, but relatively uncommon low-order operators like $\left(J_{a} J_{b}+J_{b} J_{a}\right),\left(J_{a} J_{c}+J_{c} J_{a}\right) \sin 3 \alpha,\left(J_{b} J_{c}+J_{c} J_{b}\right) \sin 3 \alpha,(1-\cos 3 \alpha) J_{b} P_{\alpha}$, $\sin 3 \alpha J_{c} P_{a}$, etc. Such fits successfully predicted a number of new E species lines. One of our better fits using RAM36 contained 174 A species lines and $228 \mathrm{E}$ species lines, with 40 adjustable parameters, and rms residuals of $8.8 \mathrm{kHz}$ and $21.4 \mathrm{kHz}$ for $\mathrm{A}$ and E species lines, respectively. The residuals from this fit are given in Table S-5 together with those from the BELGI-Cs fit of the earlier smaller data set. The complete parameter set from the RAM36 fit (in the RAM system) is available in Table S6. The RAM36 residuals are still 5 times (A species lines) and 10 times (E species lines) larger than measurement error. No amount of further fitting allowed us to reduce these residuals significantly. This then caused us to wonder if pinacolone may not have perfect $\mathrm{C}_{s}$ symmetry at equilibrium (as predicted, of course, by our initial quantum chemical calculations).

\subsubsection{BELGI-C 1 fits}

We therefore turned to BELGI-C $_{1}$ [23] (again available on PROSPE [21]). This program contains most of the torsion-rotation interaction terms of BELGI-C $\mathrm{C}_{\mathrm{s}}$, but also has nearly 30 additional terms, which are symmetry-forbidden in molecules with a plane of symmetry at equilibrium, but which become allowed when this plane is not present. One of our good fits of transitions with $J \leq 5$ and 32 adjustable parameters gives rms residuals of 12 and $13 \mathrm{kHz}$ for $72 \mathrm{~A}$ and $102 \mathrm{E}$ lines, respectively, but when a larger data set with $J \leq 7$ is fit, the residuals rise to 86 and $130 \mathrm{kHz}$ for 33 adjustable parameters (including seven allowed only for $\mathrm{C}_{1}$ symmetry at equilibrium), and $124 \mathrm{~A}$ and $174 \mathrm{E}$ lines, respectively. Some parameters from this fit are given in Table 1. Further fitting attempts with BELGI- $\mathrm{C}_{1}$ failed to reduce these residuals significantly.

\subsection{Discussion of the various fits}

Because of the extensive interlocking network of combination difference loops, as shown in Fig. 3, the $J_{\text {KaKc }}$ rotational assignments of the E species lines must, except for a very few $J=9$ and 10 lines, all be correct. While we do not have as complete an interlocking web of A loops, the A lines, apart from one or two exceptions, can be fit to $2 \mathrm{kHz}$ using a traditional asymmetric-rotor Hamiltonian (see below). Therefore, it seems reasonable to assume that the fitting problems described above arise either from "operator error" in carrying out the fits, or from some inadequacy in the theoretical models themselves. While 
the problem of operator error can never be ruled out completely, we turn now to a search for possible inadequacies in the models.

The theoretical model upon which the programs BELGI and RAM36 are based has proven to be quite satisfactory in other published work. For example, when BELGI-C $\mathrm{s}_{\mathrm{s}}$ was applied to the $160 \mathrm{~A}$ lines and $197 \mathrm{E}$ lines in a Fourier-transform jetcooled spectrum of conformer I of ethylacetamidoacetate with $J \leq 20$ and $K_{a} \leq 6$, only 15 parameters were needed to give rms residuals of $1.8 \mathrm{kHz}$ for both the $\mathrm{A}$ and $\mathrm{E}$ species lines, corresponding to 23.8 lines/parameter and a fit to experimental measurement error [24]. Similarly, when BELGI-C $_{1}$ was applied to 165 A lines and 203 E lines of conformer II of ethylacetamidoacetate, again with $J \leq 20$ and $K_{a} \leq 6$, only 18 parameters were needed to give rms residuals of $1.6 \mathrm{kHz}$ for A species and $1.8 \mathrm{kHz}$ for E species lines, corresponding to 20.4 lines/parameter [24]. In contrast, the pinacolone fit using RAM36 that was selected for deposit in the supplementary material had 10.1 lines/parameter, and gave a fit to $9 \mathrm{kHz}$ for $174 \mathrm{~A}$ lines

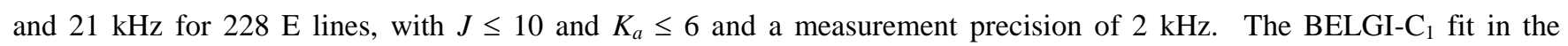
supplementary material is even worse, with 9.0 lines/parameter, and a fit to $86 \mathrm{kHz}$ for $124 \mathrm{~A}$ lines and $130 \mathrm{kHz}$ for $174 \mathrm{E}$ lines, with $J \leq 7$ and $K_{a} \leq 6$.

A similar problem occurred in our XIAM fit, where a surprisingly high standard deviation of over $1 \mathrm{MHz}$ was observed for $160 \mathrm{~A}$ and $41 \mathrm{E}$ species lines and no further predicted $\mathrm{E}$ lines could be found. We noticed that many $\mathrm{E}$ species lines, which should be correctly assigned because they can be checked by Ritz cycles, are off by up to $7 \mathrm{MHz}$ (see Table S-2). This problem was not observed previously in other molecules with an acetyl methyl internal rotor studied in Aachen. A two-top fit, considering the tert-butyl group as the second rotor and taking into account some coupling terms like $F_{12}$, did not improve the fit quality.

After the RAM36 fit was finalized, the data set from the RAM36 fit was put back in the program XIAM. This XIAM fit had a standard deviation of 4.9 MHz for $174 \mathrm{~A}$ and $228 \mathrm{E}$ transitions. Many E species lines and even some A species lines in this fit are off by more than $10 \mathrm{MHz}$. In particular, three $J=10$ transitions have a deviation of approximately $55 \mathrm{MHz}$. It thus became clear why no new E species lines could be found using the XIAM predictions. This was surprising, since from our experience, the spectra of internal-rotor molecules with barriers of approximately $100 \mathrm{~cm}^{-1}$ cannot be fitted to experimental accuracy with the program XIAM, but the predictions are often sufficient to find new lines.

Because of these unfavorable comparisons with past spectral fitting performance, we were forced to consider the possibility that the pinacolone spectrum might be perturbed in some way. Ordinarily, this would be highly unlikely for a jet- 
cooled ground state microwave spectrum, but we noticed almost from the beginning, that a nearly complete set of relatively strong unassigned lines seemed to be present in the scanned regions of the spectrum [16]. The bulk of these lines remain unassigned, even after the thorough fitting and assignment efforts reported here. Further support for the idea of some sort of perturbation (or other model error) is provided by the very poor predictive power of our fits when adding additional lines to Especies branches already in the fit. Even though the fit was good to a few tens of kHz, many of the new lines in the calculated spectrum were out by more than $1 \mathrm{MHz}$.

In an effort to shed more light on the nature of this possible perturbation, we carried out a series of asymmetric-rotorlike fits of only our A species lines, where the $J_{\max }$ value of the data set and the highest order of rotational constants used were both gradually increased. These results are summarized in Table 2, from which three conclusions can be drawn. (i) The A species lines for any value of $J_{\max }$ can be fit to $2 \mathrm{kHz}$ by going to sufficiently high order in the asymmetric rotor Hamiltonian. This fact suggests that any random local perturbations of the A species lines have magnitudes less than a few kHz. (ii) The order required for a given $J_{\max }$ is significantly higher than normal. This fact suggests that some sort of global perturbation of the A lines is present, whose effects can be mimicked by a high-order asymmetric rotor Hamiltonian. (iii) An estimate of the magnitude of this perturbation can be obtained by first noting that lines/parameter ratios of about 6 or 7 are needed to achieve $2 \mathrm{kHz}$ fits for pinacolone in the present work. If we assume that a more reasonable ratio of lines/parameter would be near 20 , then the rms of the fits in the last three rows of Table 2 suggest that the magnitude of the global perturbations are of the order of a few hundred kHz. The question now arises of where such a global perturbation might come from.

As mentioned, it is very probable from our experimental observations that some state other than the ground state is populated in our $2 \mathrm{~K}$ jet-cooled molecular beam. The primary support for the existence of a low-lying state just above the $\mathrm{v}_{\mathrm{t}}=$ 0 torsional ground state is the fact that after an exhaustive assignment of lines in the A and E sublevels, a considerable number of lines remained unassigned in the jet-cooled spectrum. Many of them are rather strong and it is thus unlikely that they are due to ${ }^{13} \mathrm{C}$ isotopologues or (small-amplitude) vibrationally excited states. Other conformers can also be excluded since only one stable conformer was predicted by our quantum chemical calculations. This low-lying state would then be a plausible candidate for a perturbation partner of the ground state. If we assume good thermal equilibrium in our beam, then the intensity of lines from this extra state suggests that it lies only a few $\mathrm{cm}^{-1}$ above the ground state, which in turn suggests that it is some tunneling component of a composite ground state arising from an as yet unidentified large-amplitude tunneling motion. If on the other hand, thermal equilibration in the beam is not perfect, then the state might lie as high as $20 \mathrm{~cm}^{-1}$ above the ground state, suggesting assignment to the first excited state of a nearly harmonic torsional oscillation of the heavy tert-butyl top 
attached to the other side of the $\mathrm{C}=\mathrm{O}$ group. We thus decided to calculate the $J=0$ torsional wave functions and eigenvalues of pinacolone that would arise from our quantum chemistry potential surface, in an attempt to determine which of these possibilities is more likely to be correct.

Table 2: Summary of asymmetric-rotor fits ${ }^{\mathrm{a}}$ to the A species lines of pinacolone.

\begin{tabular}{rrlrc}
\hline$J_{\text {max }}{ }^{b}$ & Lines $^{c}$ & Constants $^{\mathrm{d}}$ & Lines/Param & \\
& & & & $\mathrm{RMS}^{\mathrm{f}}$ \\
2 & 9 & $3 B+3 D$ & 1.5 & $14 \mathrm{kHz}$ \\
3 & 22 & $3 B+3 D$ & 3.7 & $437 \mathrm{kHz}$ \\
& & & & \\
3 & 22 & $3 B+5 D$ & 2.8 & $1.2 \mathrm{kHz}$ \\
4 & 44 & $3 B+5 D$ & 5.5 & $3.2 \mathrm{kHz}$ \\
5 & 72 & $3 B+5 D$ & 9.0 & $9.8 \mathrm{kHz}$ \\
& & & & \\
5 & 72 & $3 B+5 D+7 H$ & 4.8 & $1.9 \mathrm{kHz}$ \\
6 & 97 & $3 B+5 D+7 H$ & 6.5 & $2.0 \mathrm{kHz}$ \\
7 & 124 & $3 B+5 D+7 H$ & 8.3 & $3.0 \mathrm{kHz}$ \\
8 & 145 & $3 B+5 D+7 H$ & 9.7 & $6.0 \mathrm{kHz}$ \\
9 & 169 & $3 B+5 D+7 H$ & 11.3 & $13.7 \mathrm{kHz}^{\mathrm{g}}$ \\
& & & & \\
9 & 169 & $3 B+5 D+7 H+9 L$ & 7.0 & $1.7 \mathrm{kHz}^{\mathrm{g}}$ \\
10 & 176 & $3 B+5 D+7 H+9 L$ & 7.3 & $1.7 \mathrm{kHz}^{\mathrm{h}}$ \\
11 & 177 & $3 B+5 D+7 H+9 L$ & 7.4 & $1.8 \mathrm{kHz}^{\mathrm{h}}$ \\
13 & 179 & $3 B+5 D+7 H+9 L$ & 7.5 & $1.8 \mathrm{kHz}^{\mathrm{h}}$ \\
& & & & \\
5 & 72 & $3 B$ & 24.0 & $3.3 \mathrm{MHz}^{\mathrm{n}}$ \\
9 & 169 & $3 B+5 D$ & 21.1 & $158 \mathrm{kHz}^{\mathrm{g}}$ \\
11 & 177 & $3 B+5 D$ & 22.1 & $241 \mathrm{kHz}^{\mathrm{h}}$ \\
& & & &
\end{tabular}

${ }^{a}$ These "asymmetric-rotor" fits were actually carried out with RAM36, using fixed values of $F=5.2 \mathrm{~cm}^{-1}, V_{3}=99.4095 \mathrm{~cm}^{-1}, \rho=0.0119566$, and $D_{a b}=-0.0147874 \mathrm{~cm}^{-1}$ determined from a preliminary fit of A and $\mathrm{E}$ species lines with $J \leq 3$.

${ }^{\mathrm{b}}$ All available A-species lines with $J \leq J_{\max }$ were included in the fit in any given row of the table.

${ }^{\mathrm{c}}$ The total number of lines in the fit for this row of the table.

${ }^{\mathrm{d}}$ The asymmetric rotor constants used in the fit, represented by a shorthand notation in which $3 B$ means $A, B, C ; 3 D$ means $D_{J}, D_{J K}, D_{K} ; 5 D$ means $D_{J}, D_{J K}$, $D_{K}, \delta_{J}, \delta_{K} ;$ etc.

${ }^{\mathrm{e}}$ The ratio of lines per parameter in the fit, used as a measure of the power of the model.

${ }^{\mathrm{f}}$ The root-mean-square residual of the fit, used a measure of the quality of the model.

${ }^{\mathrm{g}}$ The line at $8331 \mathrm{MHz}$ is not in the fit.

${ }^{\mathrm{h}}$ The lines at 5551 and $8331 \mathrm{MHz}$ are not in the fit.

\section{Torsional wave functions and eigenvalues}

In this section, model calculations based on a two-dimensional potential energy surface depending on the torsional angles of both the methyl and tert-butyl groups are presented. These exploratory calculations suggest that an effective six-fold potential for the internal rotation of the acetyl methyl group causes the torsionally excited state $\left(\mathrm{v}_{\mathrm{t}}=1\right)$ to lie much closer to the ground torsional state $\left(\mathrm{v}_{\mathrm{t}}=0\right)$ than it normally does. If this energy separation is a few $\mathrm{cm}^{-1}$ or less, then transitions involving 
the $\mathrm{v}_{\mathrm{t}}=1$ state could cause additional lines in the spectrum, and interactions between the two torsional states could explain why an unusually large number of parameters were not able to reproduce the spectrum to experimental accuracy (see section 3.4).

\subsection{Theory for the $J=0$ levels of the two-top problem}

In tensor notation, the (classical) Hamiltonian of a rotating rigid molecule with two rigid internal rotors [25] can be written as

$H=\frac{1}{2} \vec{P}^{\dagger} I^{-1} \vec{P}+V\left(\alpha_{1}, \alpha_{2}\right)$

using the transposed generalized 5-dimensional angular momentum vector

$\hat{P}^{\dagger}=\left(P_{a}, P_{b}, P_{c}, p_{1}, p_{2}\right)$

where $P_{g}, g \in\{a, b, c\}$ are the components of the overall angular momentum of the molecule and $p_{k}, k \epsilon\{1,2\}$ are the angular momenta of the two internal rotors. $V\left(\alpha_{1}, \alpha_{2}\right)$ is the torsional potential depending on the internal rotation angles $\alpha_{1}, \alpha_{2}$. In the principal axis system, the inertia tensor is represented by the real symmetric matrix

$I=\left(\begin{array}{ccccc}I_{a} & 0 & 0 & \lambda_{a 1} I_{\alpha 1} & \lambda_{a 2} I_{\alpha 2} \\ 0 & I_{b} & 0 & \lambda_{b 1} I_{\alpha 1} & \lambda_{b 2} I_{\alpha 2} \\ 0 & 0 & I_{c} & \lambda_{c 1} I_{\alpha 1} & \lambda_{c 2} I_{\alpha 2} \\ \lambda_{a 1} I_{\alpha 1} & \lambda_{b 1} I_{\alpha 1} & \lambda_{c 1} I_{\alpha 1} & I_{\alpha 1} & 0 \\ \lambda_{a 2} I_{\alpha 2} & \lambda_{b 2} I_{\alpha 2} & \lambda_{c 2} I_{\alpha 2} & 0 & I_{\alpha 2}\end{array}\right)$

$I_{g}$ are the principal moments of inertia of the entire molecule, $I_{\alpha k}$ are the moments of inertia of the internal rotors, and $\lambda_{g k}$ are the direction cosines between the internal rotor axes and the principal axes of inertia. The rows and columns of the inertia tensor and also of its inverse are labeled by $a, b, c, 1,2$. The inverse inertia tensor is also a real symmetric matrix.

Converting the classical Hamiltonian to its quantum mechanical equivalent yields

$\widehat{H}=\widehat{H}_{r, r t}+\widehat{H}_{t}$.

$\widehat{H}_{r, r t}$ contains products of the overall angular momentum operators $\widehat{P}_{g}$ alone, as well as products of overall angular momentum operators and operators of the angular momenta of the internal rotors $\hat{p}_{k}$.

$\widehat{H}_{r, r t}=A^{\prime} \hat{P}_{a}^{2}+B^{\prime} \hat{P}_{b}^{2}+C^{\prime} \hat{P}_{c}^{2}+\frac{1}{2} \sum_{g<\bar{g}}\left(Z_{g \bar{g}}+Z_{\bar{g} g}\right)\left(\hat{P}_{g} \hat{P}_{\bar{g}}+\hat{P}_{\bar{g}} \hat{P}_{g}\right)-2 \sum_{g, k} Q_{g k} \hat{P}_{g} \hat{p}_{k}$ 
with $A^{\prime}=\frac{h}{8 \pi^{2}}\left(I^{-1}\right)_{a a}, B^{\prime}=\frac{h}{8 \pi^{2}}\left(I^{-1}\right)_{b b}, C^{\prime}=\frac{h}{8 \pi^{2}}\left(I^{-1}\right)_{c c}, Z_{g \bar{g}}=\frac{h}{8 \pi^{2}}\left(I^{-1}\right)_{g \bar{g}}, Q_{g k}=-\frac{h}{8 \pi^{2}}\left(I^{-1}\right)_{g k}$. The factor $\frac{h}{8 \pi^{2}}$ is needed to express the constants in frequency units.

The operator $\widehat{H}_{t}$ depends only on products of operators of the angular momenta of the internal rotors $\hat{p}_{k}$ and on the potential terms.

$\widehat{H}_{t}=F_{1} p_{1}^{2}+F_{2} p_{2}^{2}+F^{\prime}\left(\hat{p}_{1} \hat{p}_{2}+\hat{p}_{2} \hat{p}_{1}\right)+V\left(\alpha_{1}, \alpha_{2}\right)$

with $F_{1}=\frac{h}{8 \pi^{2}}\left(I^{-1}\right)_{11}, F_{2}=\frac{h}{8 \pi^{2}}\left(I^{-1}\right)_{22}, F^{\prime}=\frac{h}{8 \pi^{2}}\left(I^{-1}\right)_{12}$.

To obtain torsional eigenvalues and wave functions of the non-rotating molecule the Hamiltonian matrix is set up in the product basis $\left|J, K ; k_{1}, \sigma_{1}, k_{2}, \sigma_{2}\right\rangle$ of the symmetric top wave functions $|J, K\rangle$ and particle-on-a-ring wave functions $\left|k_{1}, \sigma_{1}, k_{2}, \sigma_{2}\right\rangle=$ $\frac{1}{2 \pi} e^{\left(3 k_{1}+\sigma_{1}\right) \alpha_{1}+\left(3 k_{2}+\sigma_{2}\right) \alpha_{2}}, k_{1}, k_{2} \in \mathbb{Z}, \sigma_{1}, \sigma_{2} \in\{0, \pm 1\}$. Since $\hat{P}^{2}$ commutes with the Hamiltonian and all other operators therein, the Hamiltonian matrix is block diagonal in the rotational quantum number $J$ and it is also block diagonal in the symmetry quantum numbers $\sigma_{1}, \sigma_{2}$. For the non-rotating molecule only the $J=0$ block is needed and the rows and columns of the Hamiltonian matrix can be labeled by $k_{1}$ and $k_{2}$ for a given torsional sublevel $\sigma_{1}, \sigma_{2}$. Since the diagonal matrix elements of $\widehat{P}_{g}^{2}, \widehat{P}_{g} \hat{P}_{\bar{g}}$, and $\widehat{P}_{g}$ are zero for $J=K=0$, the entire matrix element becomes $\left\langle 0,0 ; k_{1}^{\prime}, \sigma_{1}, k_{2}^{\prime}, \sigma_{2}\left|\widehat{H}_{r, r t}\right| 0,0, k_{1}, \sigma_{1}, k_{2}, \sigma_{2}\right\rangle=0$ and the $\widehat{H}_{r, r t}$ part of the Hamiltonian can be completely neglected for the non-rotating molecule.

The matrix elements of the torsional Hamiltonian $\widehat{H}_{t}$ do not depend on the overall rotation

$\left\langle 0,0 ; k_{1}^{\prime}, \sigma_{1}, k_{2}^{\prime}, \sigma_{2}\left|\widehat{H}_{t}\right| 0,0 ; k_{1}, \sigma_{1}, k_{2}, \sigma_{2}\right\rangle=\left\langle k_{1}^{\prime}, \sigma_{1}, k_{2}^{\prime}, \sigma_{2}\left|\widehat{H}_{t}\right| k_{1}, \sigma_{1}, k_{2}, \sigma_{2}\right\rangle$

To obtain the pure torsional energy eigenvalues and wave functions the matrix of $\widehat{H}_{t}$ is set up in the $\left|k_{1}, \sigma_{1}, k_{2}, \sigma_{2}\right\rangle$ basis for a given torsional sub-level $\sigma_{1}, \sigma_{2}$.

\subsection{Calculations}

\subsubsection{Potential energy surface}


The two-dimensional MP2/6-311++G(d,p) potential energy surface (PES), where the two dihedral angles $\alpha_{1}$ and $\alpha_{2}$ were varied over a grid of $10^{\circ}$ with all other parameters optimized, is shown in Fig. 4a. If one lets $\alpha_{1}$ increase from $0^{\circ}$ to $360^{\circ}$ along the horizontal line at $\alpha_{2}=120^{\circ}$, one finds a series of six minima, alternately offset slightly above and slightly below the $\alpha_{2}=120^{\circ}$ line. These are the six equivalent minima accessible to the light methyl rotor for one of the three equilibrium orientations of the heavy tert-butyl group (i.e., for the tert-butyl orientation that remains near $\alpha_{2}=120^{\circ}$ ). These minima are symmetrically, but not translationally, equivalent, since adjacent minima are separated alternately by $\Delta \alpha_{1} \approx 36^{\circ}$ and $\Delta \alpha_{1} \approx 84^{\circ}$.

Although not entirely evident from the color coding in Fig. $4 \mathrm{a}$, the closer lying adjacent minima $\left(\right.$ with $\left.\Delta \alpha_{1} \approx 36^{\circ}\right)$ are separated by a lower barrier of about $140 \mathrm{~cm}^{-1}$, while the more distant adjacent minima (with $\Delta \alpha_{1} \approx 84^{\circ}$ ) are separated by a higher barrier of $156 \mathrm{~cm}^{-1}$. This situation is illustrated in Fig. 5, where a one-dimensional "cut" of the potential surface is plotted. This cut is along an unusual zig-zag path consisting of the nearly straight lines connecting the six saddle points and minima by steepest descent trajectories.

In preparation for the two-internal-rotor calculation in the next section, the PES in Fig. 4a was parameterized by a twodimensional Fourier expansion. For simplicity, we only used Fourier basis functions in $3 \alpha$ and $6 \alpha$. The coefficients from this parameterization are given in Table 3. This model reproduces all calculated data points within $5 \%$ over the dynamic range of the potential, and it is in fact the potential surface calculated from this model that is plotted in Fig. 4.

The molecular geometry parameters needed to set up the 5-dimensional inertia tensor were also obtained from quantum chemical calculations at the MP2/6-311++G(d,p) level of theory. By inversion of the inertia tensor, the effective rotational constants of the methyl group $F_{1}$, the tert-butyl group $F_{2}$, and the kinetic interaction parameter $F^{\prime}$ were obtained. All these structural parameters are given in Table 3. 

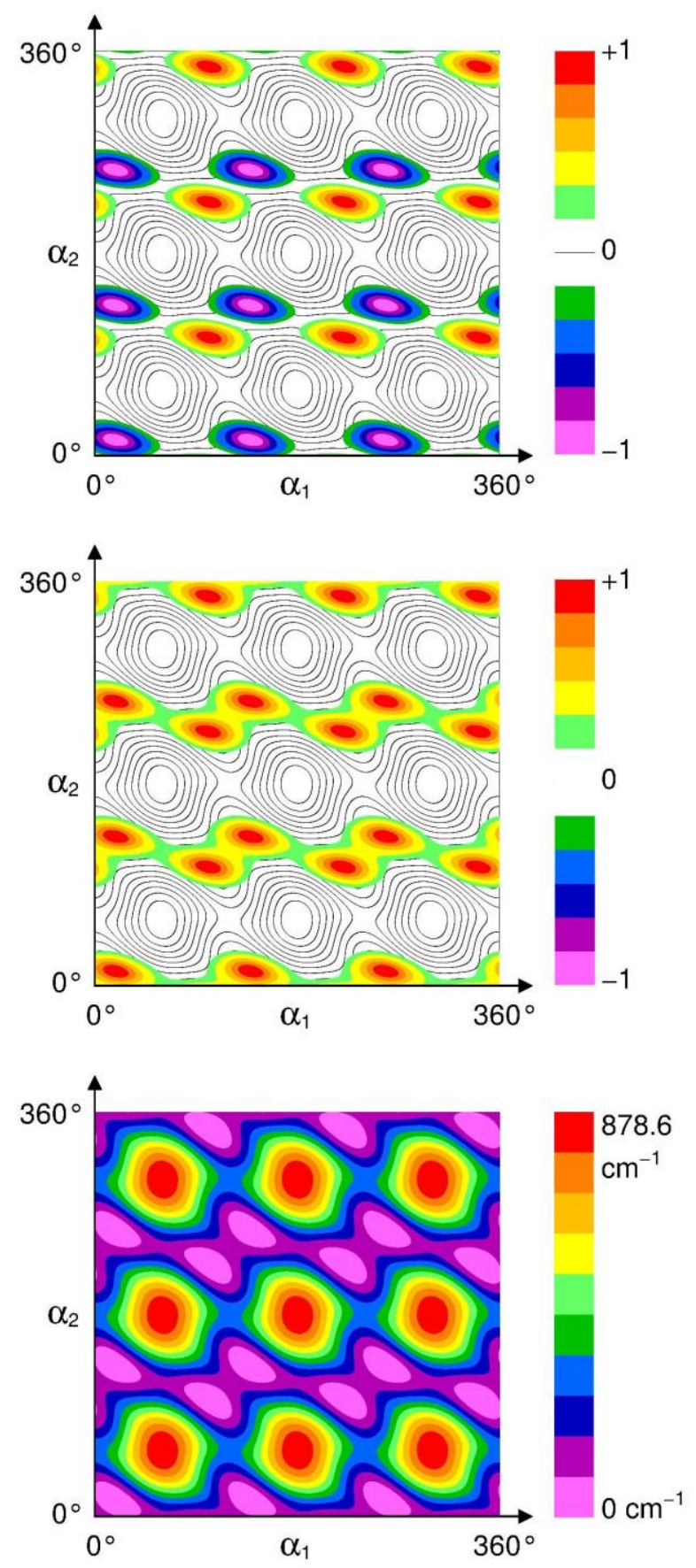

Figure 4: (a) Lower panel: The MP2/6-311++G(d,p) potential energy surface of pinacolone, as calculated with the Fourier terms given in Table 3. The angle $\alpha_{1}$ refers to rotation of the methyl top; $\alpha_{2}$ refers to rotation of the tert-butyl group. (b) Middle panel: Wave function (in arbitrary units, as indicated by the color code on the right) of the ground $\mathrm{v}=0, \sigma_{1}=\sigma_{2}=0$ state. (c) Upper panel: Wave function (in arbitrary units) of the excited $\mathrm{v}=1, \sigma_{1}=\sigma_{2}=0$ state. These three plots are most informative if the eye travels from $\alpha_{1}=0^{\circ}$ to $\alpha_{1}=360^{\circ}$ over the six minima in the acetyl methyl top internal rotation coordinate that lie alternately just above and just below the tert-butyl group orientation of $\alpha_{2}=120^{\circ}$ or $\alpha_{2}=240^{\circ}$. 
Table 3: MP2/6-311++G(d,p) parameters used for calculating the torsional wave functions and eigenvalues.

\begin{tabular}{|c|c|c|c|}
\hline Parameter $^{\mathrm{a}}$ & Unit & Value & Remarks \\
\hline$I_{a}$ & $\mathrm{u} \AA^{2}$ & 158.521458 & $A=3.188080 \mathrm{GHz}$ \\
\hline$I_{b}$ & $\mathrm{u} \AA^{2}$ & 218.317424 & $B=2.314882 \mathrm{GHz}$ \\
\hline$I_{c}$ & $\mathrm{u} \AA^{2}$ & 262.908258 & $C=1.922264 \mathrm{GHz}$ \\
\hline$I_{\alpha 1}$ & $\mathrm{u} \AA^{2}$ & 3.186191 & \\
\hline$I_{\alpha 2}$ & $\mathrm{u} \AA^{2}$ & 110.593264 & \\
\hline$\angle(a, 1)$ & $\operatorname{deg}$ & 126.255 & methyl top \\
\hline$\angle(b, 1)$ & $\operatorname{deg}$ & 143.743 & \\
\hline$\angle(c, 1)$ & $\operatorname{deg}$ & 89.662 & \\
\hline$\angle(a, 2)$ & $\operatorname{deg}$ & 8.238 & tert-butyl top \\
\hline$\angle(b, 2)$ & $\operatorname{deg}$ & 98.230 & \\
\hline$\angle(c, 2)$ & $\operatorname{deg}$ & 89.636 & \\
\hline \multicolumn{4}{|l|}{ Derived parameters } \\
\hline$F_{1}$ & $\mathrm{GHz}$ & 163.189943 & methyl top \\
\hline$F_{2}$ & $\mathrm{GHz}$ & 15.097149 & tert-butyl top \\
\hline$F^{\prime}$ & $\mathrm{GHz}$ & -5.370209 & \\
\hline Potential parameters & & & Fourier term \\
\hline$a_{1}$ & $\mathrm{E}_{\mathrm{h}}$ & 0.0015900 & 1 \\
\hline$a_{2}$ & $\mathrm{E}_{\mathrm{h}}$ & -0.0010649 & $\cos \left(3 \alpha_{1}\right)$ \\
\hline$a_{3}$ & $\mathrm{E}_{\mathrm{h}}$ & -0.0009040 & $\cos \left(3 \alpha_{2}\right)$ \\
\hline$a_{4}$ & $\mathrm{E}_{\mathrm{h}}$ & 0.0000107 & $\cos \left(6 \alpha_{1}\right)$ \\
\hline$a_{5}$ & $\mathrm{E}_{\mathrm{h}}$ & 0.0000434 & $\cos \left(6 \alpha_{2}\right)$ \\
\hline$a_{6}$ & $\mathrm{E}_{\mathrm{h}}$ & 0.0006270 & $\cos \left(3 \alpha_{1}\right) \cos \left(3 \alpha_{2}\right)$ \\
\hline$a_{7}$ & $\mathrm{E}_{\mathrm{h}}$ & -0.0004075 & $\sin \left(3 \alpha_{1}\right) \sin \left(3 \alpha_{2}\right)$ \\
\hline$a_{8}$ & $\mathrm{E}_{\mathrm{h}}$ & 0.0002462 & $\cos \left(6 \alpha_{1}\right) \cos \left(3 \alpha_{2}\right)$ \\
\hline$a_{9}$ & $\mathrm{E}_{\mathrm{h}}$ & -0.0002676 & $\sin \left(6 \alpha_{1}\right) \sin \left(3 \alpha_{2}\right)$ \\
\hline$a_{10}$ & $\mathrm{E}_{\mathrm{h}}$ & 0.0000286 & $\cos \left(3 \alpha_{1}\right) \cos \left(6 \alpha_{2}\right)$ \\
\hline$a_{11}$ & $\mathrm{E}_{\mathrm{h}}$ & -0.0000458 & $\sin \left(3 \alpha_{1}\right) \sin \left(6 \alpha_{2}\right)$ \\
\hline$a_{12}$ & $\mathrm{E}_{\mathrm{h}}$ & 0.0000706 & $\cos \left(6 \alpha_{1}\right) \cos \left(6 \alpha_{2}\right)$ \\
\hline$a_{13}$ & $\mathrm{E}_{\mathrm{h}}$ & -0.0000693 & $\sin \left(6 \alpha_{1}\right) \sin \left(6 \alpha_{2}\right)$ \\
\hline
\end{tabular}

${ }^{\text {a }}$ Structural parameters were calculated at the equilibrium geometry; potential parameters were calculated as described in Section 4.2.1.

\subsubsection{Wave functions for low-lying torsional states}

The Hamiltonian matrix of $\widehat{H}_{t}$ was set up separately for each torsional sublevel $\sigma_{1}, \sigma_{2}$. The size of the matrix is given by $\left(2 k_{1, \max }+1\right)\left(2 k_{2, \max }+1\right)$. We found that for $k_{1, \max }=k_{2, \max }=20$ convergence within $1 \mathrm{kHz}$ was achieved. The lowest torsional energies are given in Table 4 . They are only due to the methyl group $\left(\sigma_{1}\right)$. No extra splittings (above the kHz level) occur from the internal rotation of the tert-butyl group. If degeneracies associated with $\alpha_{2}$ are ignored, then energy levels are non-degenerate for $\sigma_{1}=0$ and doubly degenerate for $\sigma_{1}=1$. Therefore, it is still possible to use the convenient local mode notation $\mathrm{v} \Gamma, \mathrm{v} \in \mathbb{N}_{0}, \Gamma \in\{\mathrm{A}, \mathrm{E}\}$. Interestingly, the four energy levels $0 \mathrm{~A}, 0 \mathrm{E}, 1 \mathrm{E}, 1 \mathrm{~A}$ are found within a range of $82 \mathrm{GHz}$. 
Therefore, they are all populated under our molecular beam conditions. The higher levels are above $1220 \mathrm{GHz}$ with respect to 0A. They are unlikely to be seen in the molecular beam.

Table 4: Torsional energy levels as calculated ${ }^{a}$ with the parameters given in Table 3.

\begin{tabular}{llrrr}
\hline $\mathrm{v}$ & $\left(\sigma_{1}, \sigma_{2}\right)$ & $\mathrm{v} \Gamma$ & $v_{\mathrm{abs}} / \mathrm{GHz}$ & \multicolumn{1}{c}{$v_{\text {rel }} / \mathrm{GHz}$} \\
\hline 0 & $(0,-1),(0,0),(0,1)$ & $0 \mathrm{~A}$ & 2798.740210 & 0.000000 \\
0 & $(1,-1),(1,0),(1,1),(-1,-1),(-1,0),(-1,1)$ & $0 \mathrm{E}$ & 2816.836277 & 18.096067 \\
1 & $(1,-1),(1,0),(1,1),(-1,-1),(-1,0),(-1,1)$ & $1 \mathrm{E}$ & 2868.488492 & 69.748282 \\
1 & $(0,-1),(0,0),(0,1)$ & $1 \mathrm{~A}$ & 2880.163009 & 81.422799 \\
2 & $(0,0)$ & $2 \mathrm{~A}$ & 4019.644470 & 1220.904260 \\
2 & $(0,-1),(0,1)$ & $2 \mathrm{~A}$ & 4019.644474 & 1220.904264 \\
2 & $(1,0),(-1,0)$ & $2 \mathrm{E}$ & 4075.154577 & 1276.414367 \\
2 & $(1,1),(-1,-1)$ & $2 \mathrm{E}$ & 4075.154579 & 1276.414369 \\
2 & $(1,-1),(-1,1)$ & $2 \mathrm{E}$ & 4075.154582 & 1276.414372 \\
3 & $(1,-1),(-1,1)$ & $3 \mathrm{E}$ & 4238.112114 & 1439.371904 \\
3 & $(1,1),(-1,-1)$ & $3 \mathrm{E}$ & 4238.112119 & 1439.371909 \\
3 & $(1,0),(-1,0)$ & $3 \mathrm{E}$ & 4238.112123 & 1439.371913 \\
3 & $(0,-1),(0,1)$ & $3 \mathrm{~A}$ & 4317.514483 & 1518.774272 \\
3 & $(0,0)$ & $3 \mathrm{~A}$ & 4317.514494 & 1518.774284 \\
\hline \hline
\end{tabular}

\footnotetext{
${ }^{a}$ Calculations converged within $1 \mathrm{kHz}$ at $\mathrm{m}_{\max }=60$. Sample calculation at $\mathrm{m}_{\max }=75$ yielded the same energies. Column headings: v main torsional quantum number, $\left(\sigma_{1}, \sigma_{2}\right)$ torsional substates, $\sigma_{1}$ refers to the methyl group, $\sigma_{2}$ to the tert-butyl group, $v \Gamma$ torsional symmetry species in the local mode $C_{3 v}$ notation of the methyl group, $v_{\text {abs }}$ absolute energy level, $v_{\text {rel }}$ energy level with respect to the lowest torsional state
}

The nature of the four lowest levels is immediately clear by inspection of the corresponding wave functions. In Figs. $4 \mathrm{~b}$ and $4 \mathrm{c}$, respectively, the wave function of the $\mathrm{v}=0, \sigma_{1}=\sigma_{2}=0$ ground state (belonging to $0 \mathrm{~A}$ ) and the $\mathrm{v}=1, \sigma_{1}=\sigma_{2}=0$ excited state (belonging to $1 \mathrm{~A}$ ) is shown along with the potential energy surface. The minimum-energy path of the rotation of the methyl group consists of three equivalent double-minima. The $\mathrm{v}=0$ and the $\mathrm{v}=1$ states represent the symmetric and antisymmetric tunneling states, respectively, within each local double minimum potential. These states are combined with the A and E states arising from internal rotation of the methyl group, yielding four low-lying energy levels.

A second way of qualitatively understanding the four lowest energy levels is illustrated in Fig. 5. Here, the energy levels are plotted in the one-dimensional six-fold well that arises along the zig-zag path passing through the six minima. The energy separations of 18,52 , and $12 \mathrm{GHz}$ calculated from the two-rotor model are not too far from the 1:2:1 ratio expected for the lowest lying $\mathrm{A}_{1}, \mathrm{E}_{1}, \mathrm{E}_{2}, \mathrm{~B}_{1}$ states of one rotor in a symmetrical six-fold barrier of the form (1/2) $V_{6} \cos 6 \alpha_{1}$. 

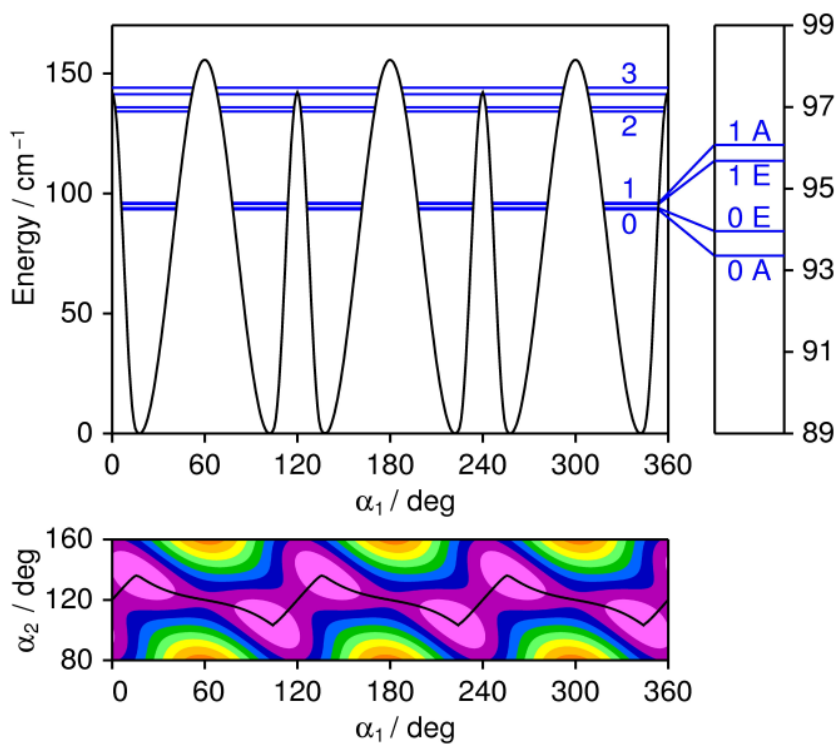

Figure 5: A one-dimensional "cut" of the relaxed potential surface in Fig. 4 along an unusual zig-zag path consisting of nearly straight lines connecting the six saddle points and minima by steepest descent trajectories, as indicated in the lower panel of the figure (color code as in Fig. 4). The horizontal axis does not measure distance along this path, but instead gives of the acetyl methyl top torsional angle $\alpha_{1}$ along the path. The closer lying adjacent minima (with $\Delta \alpha_{1} \approx 36^{\circ}$ ) are separated by a lower barrier of $140 \mathrm{~cm}^{-1}$, while the more distant adjacent minima (with $\Delta \alpha_{1} \approx 84^{\circ}$ ) are separated by a higher barrier of $156 \mathrm{~cm}^{-1}$. Energy levels from a two-top calculation (see text) are plotted in the potential curve. Each of the $v=0,1,2$, and 3 levels has an A and an E component, corresponding to internal rotation splittings of a methyl top in a three-fold barrier, but the spacings of these splittings are reminiscent of those for a methyl top in a six-fold barrier. This splitting pattern and the zig-zag path connecting the six minima in Fig. 5 suggests that the large-amplitude-motion problem in pinacolone might be converted into a one-dimensional six-fold well problem (see text).

\section{Discussion}

Molecular parameters from the various fits carried out in this work are collected in Table 1. The $V_{3}$ potential of the acetyl methyl group in pinacolone was determined by XIAM from combined A and E species lines to be 121.532(57) $\mathrm{cm}^{-1}$, but if results from other fits are considered, this parameter is seen to vary over a range of $30 \mathrm{~cm}^{-1}$. While some variation in parameters is expected for different fitting programs, the variation in Table 1 is unreasonably large. As discussed below, we believe this variation arises because the internal dynamics of this molecule are not correctly captured by any of the presently existing torsion-rotation fitting programs. Further support for this hypothesis comes from the large number of centrifugal distortion constants needed for the XIAM fit of A species lines (Fit A in Table 1), which is quite unusual for a fit of only low- $J$ transitions (i.e., $J \leq 10)$. 
We turn now to what we believe is the most important question raised by the present work, namely: What underlying physics is missing from the treatment of torsion-rotation dynamics in presently existing computer programs? Also, once this missing physics is identified, how might the inadequacy be rectified so that a large-amplitude-motion problem of the type presented by pinacolone can be quantitatively understood?

The two-rotor pure torsional calculations in Section 4 (which were carried out after all attempts to obtain a good fit with existing torsion-rotation programs had been exhausted) quite successfully explain the large number of strong unassigned lines that remain in our pinacolone spectrum by attributing them to transitions within the $\mathrm{E}$ and $\mathrm{A}$ components of the first excited torsional state of the acetyl methyl top, as shown in Figs. $4 \mathrm{c}$ and 5. This suggests that use of a two-inequivalent-top program like BELGICs-2tops [26] might be one way of carrying out a global fit of all microwave transitions observed in the jet-cooled spectrum of pinacolone. Two possible sources of trouble in this approach will be the need for a numerically adequate treatment of the extremely large interaction terms between the methyl and tert-butyl internal rotation motions in the potential energy function (associated with our parameters $a_{6}$ to $a_{13}$ in Table 3), as well as a numerically adequate treatment of the extremely large momentof-inertia imbalance between the methyl and tert-butyl tops in the kinetic energy operator. A third source of trouble arises from the fact that the heavy tert-butyl top does not actually undergo a full $2 \pi$ internal rotation, so it does not sense regions of the potential function between each of the three horizontal strips of six minima shown in Fig. 4. For this reason, it will be nearly impossible to determine experimentally potential constants describing this region (e.g., the second barrier height in the two-inequivalent-top model) by fitting observed transitions to energy-level calculations from this model. Also for this reason, it is not useful to treat the pinacolone spectrum using a $\mathrm{G}_{18}$ permutation-inversion group, since oscillations of the tert-butyl group are not large enough to make tunneling through an angle of $120^{\circ}$ feasible (see Fig. 4).

The zig-zag path connecting the six minima in Fig. 5 suggests that the large-amplitude-motion problem in pinacolone might be converted into a one-dimensional problem by defining a coordinate $s$ that measures distance along this path, which would then permit a traditional one-top internal rotation program to be a second way of carrying out a global fit. Two interesting and rather general theoretical questions associated with this approach come to mind immediately. First, how should one deal quantum mechanically with the very large changes in the classical momentum vector that arise from the near-zero radius of curvature at the corners of one-dimensional zig-zag paths? Dealing with these sharp corners will probably be the main difficulty in trying to apply a one-dimensional formalism to this problem. Second, can further study of a large-amplitude motion that tunnels back and forth six-times between chiral conformers before returning to its starting configuration lead to new experiments or new insights into our understanding of chirality? 
Finally, the six minima near $\alpha_{2}=120^{\circ}$ in Fig. 4 a are very similar to the six minima in the two-dimensional potential energy surfaces describing the $\mathrm{NH}_{2}$ inversion and $\mathrm{CH}_{3}$ internal-rotation motions in $\mathrm{CH}_{3} \mathrm{NH}_{2}$ [27] or describing the $\mathrm{CH}_{2}$ inversion and $\mathrm{CH}_{3}$ internal rotation in $\mathrm{CH}_{3} \mathrm{CH}_{2}$ [28]. The splittings calculated in Section 4 for the first four $0 \mathrm{~A}, 0 \mathrm{E}, 1 \mathrm{E}$, and $1 \mathrm{~A}$ states in pinacolone are relatively small, suggesting that a tunneling treatment similar to that used for methylamine [29] might be a third possibility for carrying out a global fit. In such a treatment, the methyl top motion would be treated as an internal rotation, while the motion of the tert-butyl group would be treated as a back-and-forth oscillation. A fourth possibility would be a semirigid-model treatment similar to that used for the ethyl radical [28]. Possible complications for both models in this paragraph would include dealing with the symmetrical inequivalence of the three long and three short tunneling paths in Fig. 5.

Before attempting more global fits, however, it is almost essential to carry out the following experimental studies. (i) Make a complete scan of the pinacolone spectrum in the region from 2 to $40 \mathrm{MHz}$ accessible with the two Aachen spectrometers. This should be easily possible, since continuous scans already exist for small regions of the spectrum. (ii) Look for Ritz cycles in a spectrum from which all ground-state lines have been removed, in order to get a start on rotational assignments for the remaining lines. We have in fact already found one or two promising candidates for Ritz cycles in these lines, but confirmation is not possible because of the absence of complete scans. (iii) Use one or more of the theoretical approaches mentioned above to attempt global fits of the measured spectra.

Another promising set of new experiments would involve full deuteratation of one, the other, and then both of the $\mathrm{C}_{3 \mathrm{v}}$ methyl and tert-butyl groups. The high-resolution spectroscopy will be degraded significantly because of the quadrupole hyperfine structure associated with three, nine, or twelve deuterium atoms in the molecule, but increasing the moment of inertia of one or both functional groups will certainly shed light on the degree of cooperative motion involved, and thus permit an assessment of the current quantum chemical calculations. The latter, for example, may give a correct description of the delicate balance of forces in pinacolone only when carried out at a much higher level than used here. 


\section{Acknowledgments}

The authors are greatly indebted to Dr. Isabelle Kleiner and Dr. Vadim Ilyushin for significant help in the early stages of using their programs BELGI-C $\mathrm{C}_{\mathrm{s}}$, BELGI- $\mathrm{C}_{1}$, and RAM36. The authors also thank Dr. Peter Groner for making the program ERHAM available to the spectroscopic community. The IT center of the RWTH Aachen University, the Land NordrheinWestfalen, and the Theodor von Kármán fellowship of the RWTH Aachen University program are greatly acknowledged for free computer time and for funds.

\section{References}

[1] H. Mouhib, W. Stahl, Chem. Phys. Chem. 13 (2012) 1297.

[2] L. W. Sutikdja, D. Jelisavac, W. Stahl, I. Kleiner, Mol. Phys. 110 (2012) 2883.

[3] T. Attig, R. Kannengiesser, I. Kleiner, W. Stahl, J. Mol. Spectrosc. 290 (2013) 24.

[4] H.V. L. Nguyen, A. Jabri, V. Van, W. Stahl, J. Phys. Chem. A 118 (2014) 12130.

[5] H.V. L. Nguyen, W. Stahl, Chem. Phys. Chem. 12 (2011) 1900.

[6] Y. Zhao, W. Stahl, H. V. L. Nguyen, Chem. Phys. Lett. 545 (2012) 9.

[7] Y. Zhao, J. Jin, W. Stahl, I. Kleiner, J. Mol. Spectrosc. 281 (2012) 4.

[8] A. C. Fantoni, W. Caminati, R. Meyer, Chem. Phys. Lett. 133 (1987) 27.

[9] B. Velino, A. Maris, S. Melandri, W. Caminati, J. Mol. Spectrosc. 256 (2009) 228.

[10] W. Stahl, H. Dreizler, M. Hayashi, Z. Naturforsch. 38a (1983) 1010.

[11] M. J. Frisch, G. W. Trucks, H. B. Schlegel, G. E. Scuseria, M. A. Robb, J. R. Cheeseman, G. Scalmani, V. Barone, B. Mennucci, G. A. Petersson, H. Nakatsuji, M. Caricato, X. Li, H. P. Hratchian, A. F. Izmaylov, J. Bloino, G. Zheng, J. L. Sonnenberg, M. Hada, M. Ehara, K. Toyota, R. Fukuda, J. Hasegawa, M. Ishida, T. Nakajima, Y. Honda, O. Kitao, H. Nakai, T. Vreven, J. A. Montgomery, Jr., J. E. Peralta, F. Ogliaro, M. Bearpark, J. J. Heyd, E. Brothers, K. N. Kudin, V. N. Staroverov, R. Kobayashi, J. Normand, K. Raghavachari, A. Rendell, J. C. Burant, S. S. Iyengar, J. Tomasi,M. Cossi, N. Rega, J. M. Millam, M. Klene, J. E. Knox, J. B. Cross, V. Bakken, C. Adamo, J. Jaramillo, R. Gomperts, R. E. Stratmann, O. Yazyev, A. J. Austin, R. Cammi, C. Pomelli, J. W. Ochterski, R. L. Martin, K. Morokuma, V. G. Zakrzewski, G. A. Voth, P. Salvador, J. J. Dannenberg, S. Dapprich, A. D. Daniels, O. Farkas, J. B. Foresman, J. V. Ortiz, J. Cioslowski and D. J. Fox, Gaussian 09, Revision A.02, Gaussian, Inc., Wallingford CT, 2009.

[12] Certain commercial products are identified in this paper in order to specify adequately the experimental or theoretical procedures. In no case does such identification imply recommendation or endorsement by the National Institute of Standards and Technology, nor does it imply that the products are necessarily the best available for the purpose.

[13] J.-U. Grabow, W. Stahl, H. Dreizler, Rev. Sci. Instrum. 67 (1996) 4072.

[14] I. Merke, W. Stahl, H. Dreizler, Z. Naturforsch. 49a (1994) 490. 
[15] B. N. Taylor, C. E. Kuyatt (1994). Guidelines for Evaluating and Expressing the Uncertainty of NIST Measurement Results. http://physics.nist.gov/TN1297

[16] Y. Zhao, Dissertation, RWTH Aachen University, 2013.

[17] H. Hartwig, H. Dreizler, Z. Naturforsch. 51a (1996) 923.

[18] J. K. G. Watson, J. Chem. Phys. 46 (1967) 1935.

[19] P. Groner, J. Chem. Phys. 107 (1997) 4483.

[20] J. T. Hougen, I. Kleiner, M. Godefroid, J. Mol. Spectrosc. 163 (1994) 559.

[21] http://www.ifpan.edu.pl/ kisiel/prospe.htm.

[22] V.V. Ilyushin, Z. Kisiel, L. Pszczółkowski, H. Mäder, J. T. Hougen, J. Mol. Spectrosc. 259 (2010) 26.

[23] I. Kleiner, J. T. Hougen, J. Chem. Phys. 119 (2003) 5505.

[24] R. J. Lavrich, A. R. Hight Walker, D. F. Plusquellic, I. Kleiner, R. D. Suenram, J. T. Hougen, G. T. Fraser, J. Chem. Phys. 119 (2003) 5497.

[25] H. Dreizler, Fortschr. Chem. Forsch. 10 (1968) 59.

[26] M. Tudorie, I. Kleiner, J. T. Hougen, S. Melandri, L. W. Sutikdja, W. Stahl, J. Mol. Spectrosc. 269 (2011) 211.

[27] M. Tsuboi, A. Y. Hirakawa, T. Ino, T. Sasaki, K. Tamagake, J. Chem. Phys. 41 (1964) 2721.

[28] M. Kręglewski, I. Gulaczyk, Chem. Phys. Lett. 592 (2014) 307.

[29] N. Ohashi, J. T. Hougen, J. Mol. Spectrosc. 121 (1987) 474. 